\title{
An investigation of laboratory-grown ice spikes
}

\author{
KenNeth G. LIBBRECHT, Kevin LUI \\ Norman Bridge Laboratory of Physics, California Institute of Technology 264-33, Pasadena, California 91125, U.S.A. \\ E-mail:kgl@caltech.edu
}

\begin{abstract}
We have investigated the formation of $10-50 \mathrm{~mm}$ long ice spikes that sometimes appear on the free surface of water when it solidifies. By freezing water under different conditions, we measured the probability of ice-spike formation as a function of: (1) the air temperature in the freezing chamber, (2) air motion in the freezing chamber (which promotes evaporative cooling), (3) the quantity of dissolved salts in the water, and (4) the size, shape and composing material of the freezing vessel. We found that the probability of ice-spike formation is greatest when the air temperature is near $-7^{\circ} \mathrm{C}$, the water is pure and the air in the freezing chamber is moving. Even small quantities of dissolved solids greatly reduce the probability of ice-spike formation. Under optimal conditions, approximately half the ice cubes in an ordinary ice-cube tray will form ice spikes. Guided by these observations, we have examined the Bally-Dorsey model for the formation of ice spikes. In this model, the density change during solidification forces supercooled water up through a hollow ice tube, where it freezes around the rim to lengthen the tube. We propose that any dissolved solids in the water will tend to concentrate at the tip of a growing ice spike and inhibit its growth. This can qualitatively explain the observation that ice spikes do not readily form using water containing even small quantities of dissolved solids.
\end{abstract}

\section{INTRODUGTION}

When water freezes into ice, the expansion that occurs during the solidification process sometimes causes the formation of ice spikes that rise out of the free ice surface. The phenomenon has been observed sporadically outdoors in cold climates for many decades (Dorsey 1921; Hallet 1960; Perry, 1993, 1995; Abrusci 1997; Knight, 1998). These rare sightings have documented the appearance of rather large ice spikes, perhaps $100 \mathrm{~mm}$ long, sometimes with triangular cross-sections, usually forming overnight in containers of standing water. Much smaller ice spikes (also known as ice spicules) have been observed coming out of sleet particles (Alty, 1933; Bally, 1933; Blanchard, 1951; Mason and Maybank, 1960). These appear in droplets of order $1 \mathrm{~mm}$ in size or larger and they have been successfully reproduced and studied in the laboratory (Blanchard, 1951; Mason and Maybank, 1960).

More recently, numerous reports have appeared on the internet describing the appearance of $10-50 \mathrm{~mm}$ long ice spikes forming when water is frozen in ordinary ice-cube trays in ordinary household freezers. (As of this writing, reports of ice spikes can be found at

\footnotetext{
$<$ http://www.physics.utoronto.ca/ smorris/edl/icespikes/ icespikes.html>,

$<$ http://www.its.caltech.edu/ atomic/snowcrystals/ icespikes/icespikes.htm $>$,

$<$ http://www.yk.psu.edu/ kxt7/IceSpikes.html $>$,

$<$ http://www.tesla-coil.org/Ice\%20Cubes.htm>,

$<$ http://members.shaw.ca/FibbWEB/> and

$<$ http://www.photodude.com/weblog/archives/

00000020.shtml>.
}

To our knowledge, the appearance of ice spikes under such circumstances has received little attention in the scientific literature (however, see Perry, 1993, 1995) and the topic has not been the subject of any systematic laboratory studies. A key element in the production of ice spikes - the purity of the water that is frozen - has only briefly been touched upon in any of the previous studies of ice spikes (Mason and Maybank, 1960). Ice spikes rarely form when tap water is frozen, but do so frequently using distilled water. This important point was discovered and communicated to us by J. F. Cooper, III (personal communication, 2002), although it appears it was independently discovered by a number of others (see internet references above). If household distilled water is used (the type sold in grocery stores), then a plastic ice-cube tray in a typical freezer will yield approximately one to four ice spikes that look like the examples shown in Figure 1. The spikes form at apparently random angles with respect to vertical and with various lengths. Occasionally more than one spike will grow out of a single cube, and we once found three spikes emanating from a common base. The longest ice spike we observed in any of our experiments using ice-cube trays was $56 \mathrm{~mm}$ from base to tip. These spikes typically have roughly circular cross-sections (sometimes with a rounded triangular cross-section near the base), and the spikes often display narrow, fairly sharp tips. Even when an ice cube does not contain a spike, the ice surface is typically not flat, but includes several mm-scale surface ice lumps. Tap water usually does not form ice spikes, although it does form ice lumps.

The generally accepted mechanism for the formation of ice spikes of all sizes is the Bally-Dorsey model (Dorsey, 1921; Bally, 1933; Blanchard, 1951). In the case of an ice spike forming in an ice-cube tray, water first freezes at the surface, starting at the edges of the cube, and the ice subsequently 

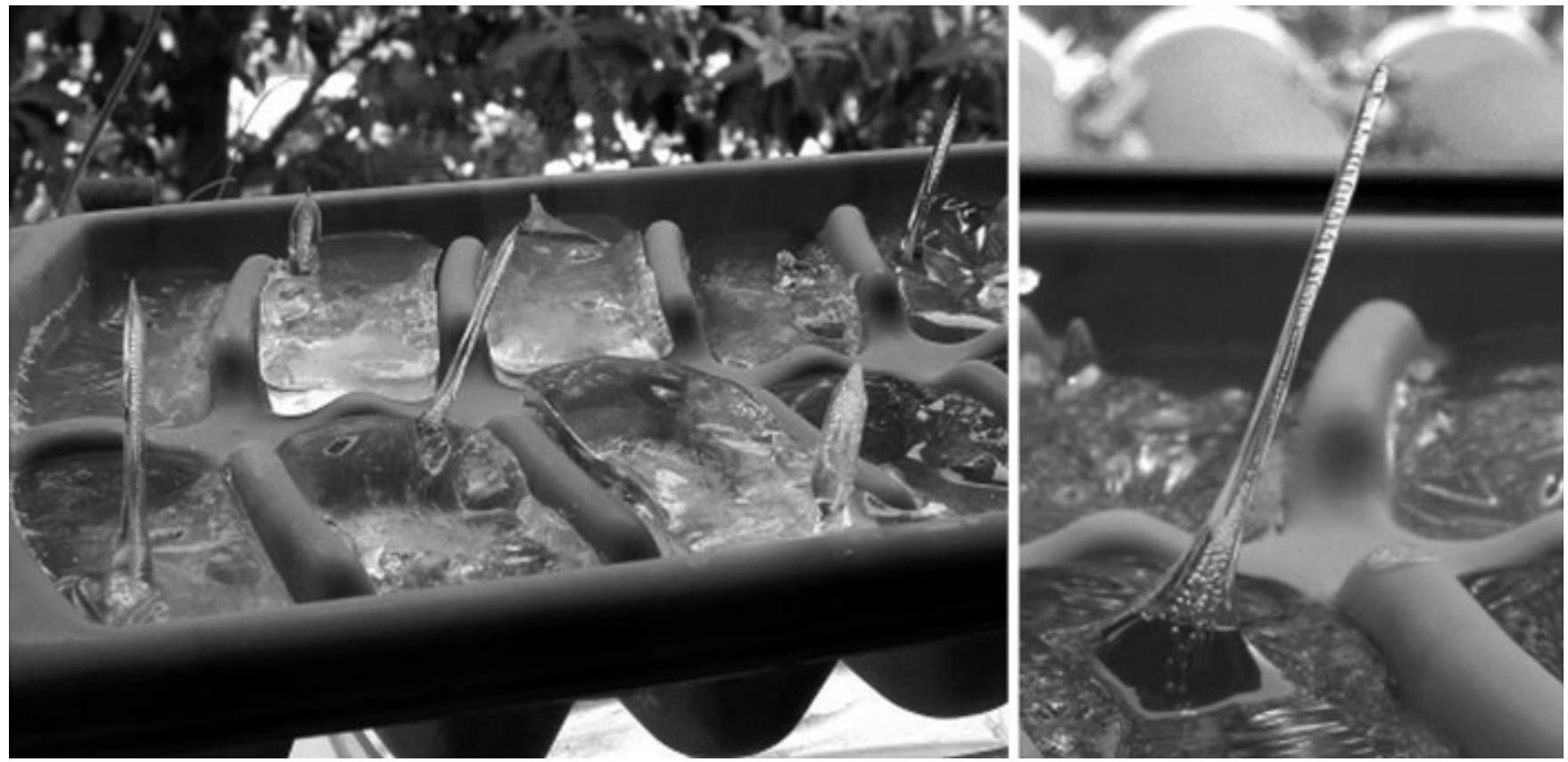

Fig. 1. A number of ice spikes grown in an ordinary plastic ice-cube tray using distilled water. Note the spikes grow at many different positions and angles and to various heights up to $\approx 50 \mathrm{~mm}$.

expands laterally until only a small hole in the ice surface remains. Then the continued freezing of water beneath the surface forces water up through the hole, where it freezes around the edge of the hole to form the beginnings of a hollow tube. Continued freezing forces water up through the tube, where it freezes around the rim and lengthens the tube. At some point the tube freezes shut and growth stops.

Below we examine ice-spike formation under a variety of different growth conditions. Guided by these observations, we have further examined the Bally-Dorsey model, in particular regarding the growth of ice spikes in the presence of dissolved solids in the water.

\section{OBSERVATIONS}

Our observations were primarily focused on measuring the

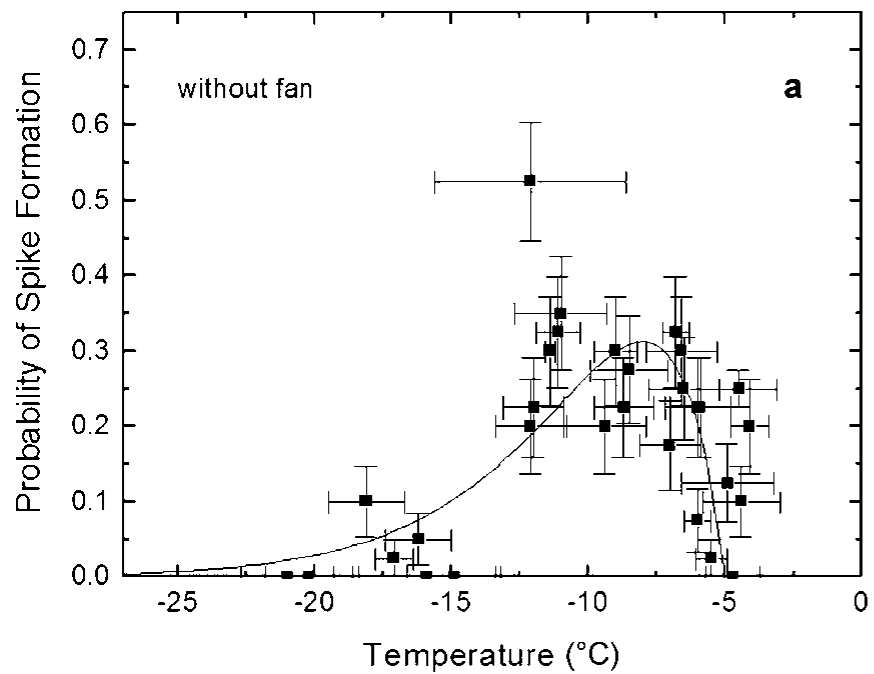

probability of ice-spike formation as a function of various growth conditions. The experiments were performed in an insulated cylindrical copper cold tank measuring $0.5 \mathrm{~m}$ in diameter by $0.6 \mathrm{~m}$ in height that was cooled by circulating cold methanol through copper tubing soldered to the sides and top of the tank. Freezing vessels, usually ordinary plastic ice-cube trays in which the volume of a single cube was $18 \mathrm{~mL}$, were placed on a steel wire rack that held the trays in the middle of the tank. For some trials a fan inside the tank circulated the air to promote evaporative cooling. The face of the fan had an area of $\approx 50 \mathrm{~cm}^{2}$, it produced an air velocity of roughly $2 \mathrm{~m} \mathrm{~s}^{-1}$ and it was either on or off. We used laboratory deionized water, without further purification, for all our trials.

Ice spikes form quickly once the water begins to freeze. We videotaped several trials using a miniature TV camera placed inside the tank and found that spikes typically grow

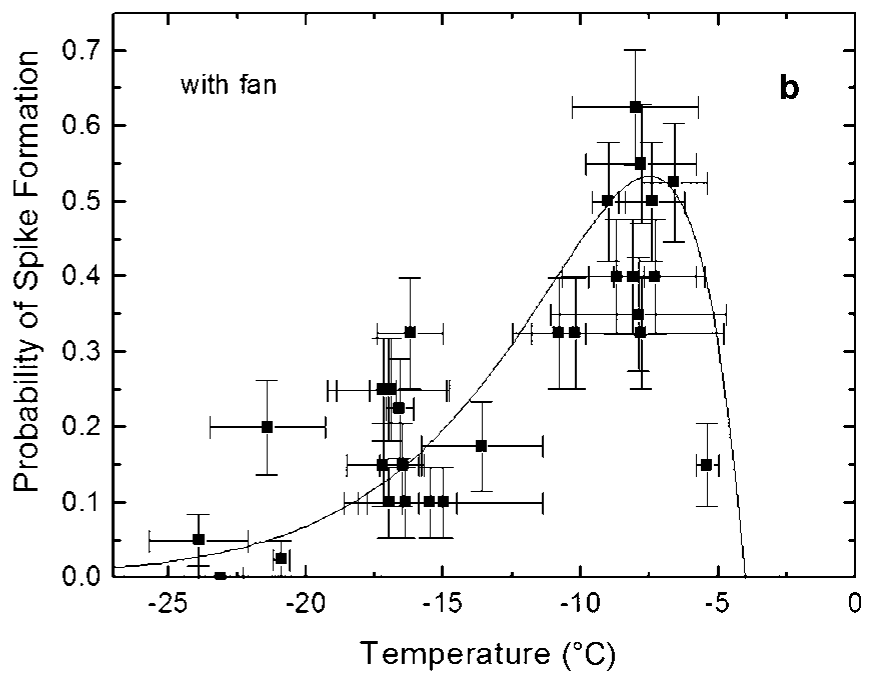

Fig. 2. Measurements of the probability that a single ice cube in a tray will produce an ice spike longer than a few millimeters. Each point refers to a single trial consisting of 40 ice cubes. The left plot is for ice cubes grown in still air, and the right plot is with a fan circulating air in the cold tank. The curves in both plots were drawn to guide the eye. 
to their full height in 3-10 min, at growth velocities of roughly $50 \mu \mathrm{m} \mathrm{s}^{-1}$. If sufficiently disturbed, some spikes were observed to change direction in the middle of their growth. The growth of a spike stops when the tube freezes shut, which happens long before the entire ice cube is frozen.

\section{Dependence on temperature and air motion}

We froze several thousand ice cubes (by which we mean those in ordinary ice-cube trays, which are not actually cubical in shape) in several dozen trials to determine the probability of ice-spike formation as a function of temperature and air motion. By probability we mean the probability that a single ice cube in an ice-cube tray will produce a spike that rises more than a few millimeters above the ice surface. We assume that all the cubes in a tray are independent of one another.

Figure 2a shows the probability as a function of temperature for still air. The error bars in temperature arise because the room-temperature water initially produced a substantial perturbation of the air temperature in the tank. The individual temperature error bars on this plot span from the initial chamber temperature (equal to the lowest temperature during the trial, also equal to the final chamber temperature) to the temperature a few minutes after the trays were placed in the chamber (the highest temperature during the trial). The displayed temperature was the average of these two values. The vertical error bars were derived assuming Bernoulli statistics. Figure 2b displays similar data, except that for these points the fan circulated air in the chamber.

The sharp decline in ice-spike formation above $-5^{\circ} \mathrm{C}$ coincided with a sudden increase in the time needed to initiate freezing that occurred at about the same temperature. Substantially below $-5^{\circ} \mathrm{C}$, it took about 2 hours for freezing to begin and the freezing time slowly increased with increasing temperature. Around $-5^{\circ} \mathrm{C}$ the freezing time increased abruptly and at temperatures above $-4^{\circ} \mathrm{C}$ the water often reached a metastable supercooled state that did not freeze for many hours.

The broad maxima seen in Figure 2 suggest that most household freezers are sufficient to produce ice spikes using distilled water, which we confirmed with additional trials using a number of freezers around the Caltech campus. Modern frost-free freezers circulate cold dry air through the freezing compartment using a fan, in contrast to older freezers that generally do not have circulating fans. The former are more likely to produce ice spikes, as we can see from the data in Figure 2.

\section{Dependence on salt concentration}

We examined how the probability of ice-spike formation varied with dissolved solids by forming solutions of ordinary table salt in deionized water at different concentrations and freezing them at a chamber temperature of $-7^{\circ} \mathrm{C}$. Figure 3 shows the ice-spike probability as a function of solution concentration. Since medium-hard tap water is typically a $\approx 10^{-3}$ molar solution of various mineral salts, we see that the data in Figure 3 are consistent with the observation that ice spikes only rarely form from tap water. Even a concentration of $10^{-5}$ molar is sufficient to reduce the ice-spike probability by a factor of two from the value for deionized water.

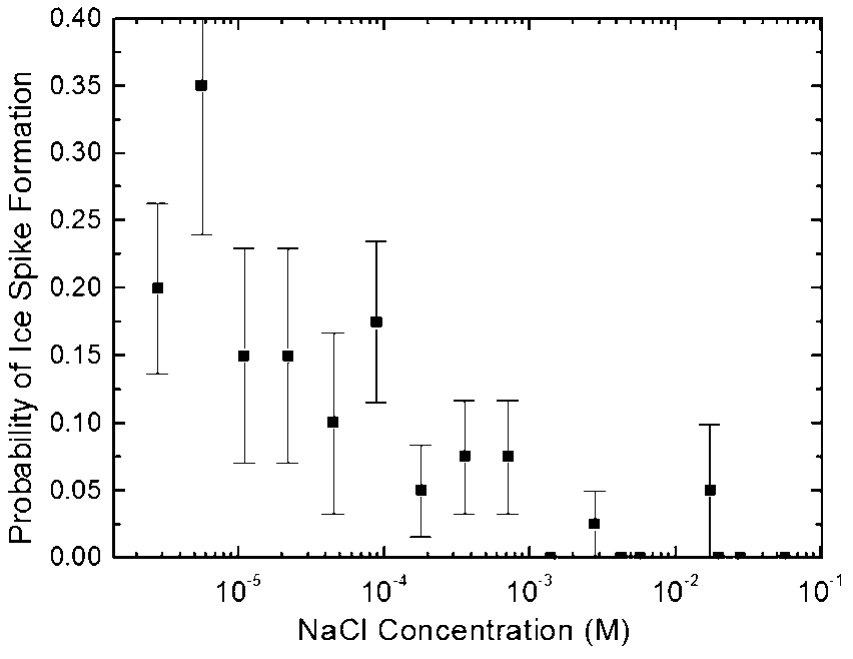

Fig. 3. Probability of ice-spike formation as a function of the solution concentration of table salt in deionized water.

\section{Dependence on container size and composition}

We performed several trials near the temperature peak (see Fig. 2) using old-fashioned aluminium ice-cube trays, after the individual cubes had been sealed using silicone caulk (to prevent the flow of water between cubes). These yielded few spikes, suggesting that the thermally insulating properties of plastic ice-cube trays are beneficial. We therefore did some additional trials using plastic ice-cube trays to which we had added additional Styrofoam insulation on the bottom. These trials indicated that the added insulation reduced the ice-spike probability by a small but significant factor when the fan was on. These observations indicate that an ordinary plastic ice-cube tray provides a near-optimal amount of thermal insulation for forming ice spikes.

We also performed several trials using cylindrical acrylic freezing vessels of different sizes, ranging from approximately 1 to $25 \mathrm{~cm}^{3}$ in volume. In these trials we observed that the larger containers had a slightly higher probability of icespike formation and that the length and girth of the ice spikes is somewhat larger in the larger containers. Ultrapure water increases the production of ice spikes in larger containers, allowing the formation of spikes up to $100 \mathrm{~mm}$ long (personal communication from M. Elgersma, 2003).

\section{DISGUSSION}

The Bally-Dorsey model for ice-spike formation is illustrated in Figure 4. If the chamber temperature is sufficiently cold ( say around $-10^{\circ} \mathrm{C}$ ), then nucleation sites are plentiful and ice first forms on the surface near the edges of the container. Ice subsequently grows in from the edges until only a small hole in the ice sheet remains unfrozen. If the freezing rate is sufficiently high beneath the surface, then the expansion that accompanies solidification will begin to force water out through the remaining hole in the ice. One frequently observes partially frozen ice cubes in which the ice surface has been wet by this process. If faceting is present in the surface ice, then the central hole may become triangular in shape, which probably explains the formation of ice spikes with triangular cross-sections.

If conditions are right, the water pushed through the hole in the ice will freeze around the edges of the hole and form a short tube of ice. Once a tube is established, water 


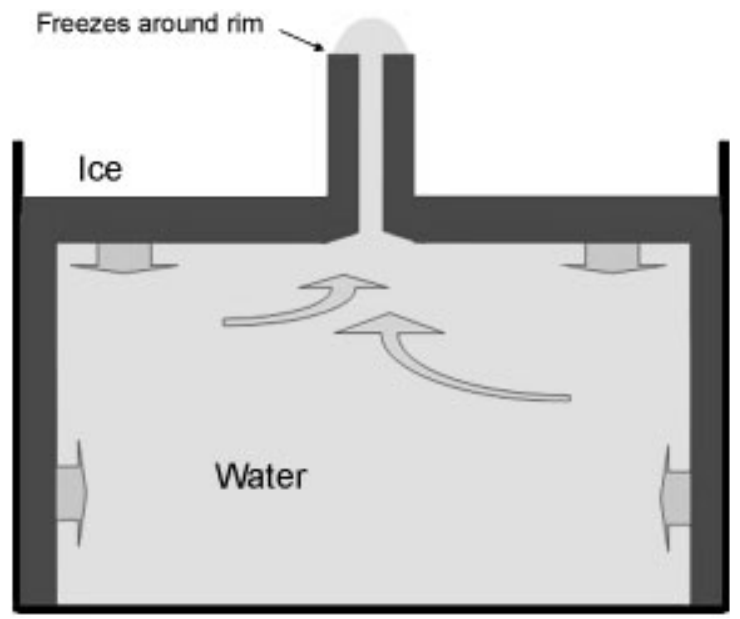

Fig. 4. The Bally-Dorsey model for ice-spike formation. Ice spikes grow as the solidification process forces water up through an ice tube, where it freezes and lengthens the tube. As described in the text, this model can explain why ice spikes form preferentially in pure water and in the presence of airflow.

forced up through the tube will form an unfrozen droplet perched atop the tube. The droplet will freeze around the edges of the tube, thus increasing the length of the tube. If a tube of the proper shape does not initially form, as is often the case, then the water forced out of the freezing cube simply spills out over the ice surface, typically forming small ice mounds on the surface.

We can see that the growth of an ice spike will be stable to small perturbations in the flow of water up the tube. If the water flow increases, then the unfrozen droplet on top of the tube will increase in size. The larger droplet has a larger surface area, so evaporative cooling will tend to increase the rate of freezing around the edges of the tube. With a large enough flow, water would spill over the sides of the tube, but ice spikes typically show no signs of this. For a reduced rate of water flowing up the tube, the droplet will shrink and the freezing rate will diminish. This suggests that, over some range of water flows, the freezing at the top of the tube will adjust to match the water flow from below. The tube will continue growing until it freezes shut somewhere along its length.

The dramatic dependence of ice-spike formation on impurities in the water likely stems from the fact that impurities are generally not incorporated into the ice lattice during the freezing process. Thus, during the formation of an ice spike, impurities will become increasingly concentrated in the small, unfrozen droplet at the top of the tube. These impurities reduce the freezing rate and so the growth of the tube. When the initial impurity rate is sufficiently high, most nascent tubes grow slowly enough that they freeze shut before reaching an appreciable length. On the rare occasions when exceptionally large spikes grow in natural, outdoor ice formations, it appears some other mechanism may be necessary to remove the impurities that build up at the top of the growing tube. The impurities may be forced into pockets that freeze more slowly, as happens with sea ice, or perhaps a convective flow replaces the water at the top of the tube with fresh water from below. Significant convection is unlikely in the smaller tubes that form in ice-cube trays. This model also provides a simple explanation for the fact that air motion enhances the production of ice spikes. Evaporative cooling will enhance the stabilization mechanism described above and it will increase the freezing rate of the droplet on top of a growing tube. Since evaporative cooling will not greatly increase the freezing rate of the inner walls of the tube, an increase in the freezing rate at the top should produce longer ice spikes.

It appears that evaporative cooling plays an important role in ice-spike formation even in still air. If evaporative cooling were absent, then conductive heat-flow considerations suggest that the ice growth rate inside the tube would not be dramatically slower than that at the top of the tube. If that were the case, then the tubes would freeze shut relatively quickly and one would not expect ice spikes that are 10-20 times longer than their diameters, as we observe.

It may also be the case that the water flow through the tubes slows the freezing rate along the tube walls by a substantial amount, inasmuch as it affects the heat flow inside the tube. We might expect such a mechanism to work less well at lower temperatures and this might explain the decrease in ice-spike formation below the peaks seen in Figure 2.

Making the Bally-Dorsey model more quantitative remains challenging. One would need to model heat flow in and around the tube, evaporative cooling from the spike tip, the degree of supercooling at various points along the tube, the effect of flow on freezing and many other factors. It should be possible, however, to manufacture ice spikes in a much more controlled manner than has been done to date. Once a method is developed to begin the ice tube, water could be forced up through the growing tube using an externally applied pressure. One could then measure the tube growth rates under different conditions to explore this fascinating phenomenon in more detail.

\section{REFERENCES}

Abrusci, G. 1997. What conditions determine crystal growth? Am. F. Phys, $65(10), 941$.

Alty, T. 1933. The maximum rate of evaporation of water. Philos. Mag., 15, 82-85.

Bally, O. 1933. Über eine eigenartige Eiskrystallbildung. Helvetica Chimica Acta, 18, 475-476.

Blanchard, D. C. 1951. A verification of the Balley-Dorsey theory of spicule formation on sleet pellets. F. Meteorol., 8(4), 268-269.

Dorsey, H. E. 1921. Peculiar ice formations. Phys. Rev., 18(2), 162-164.

Hallett, J. 1960. Crystal growth and the formation of spikes in the surface of supercooled water. F. Glaciol., 3(28), 698-704.

Knight, C. A. 1998. Answer to question \#65. What conditions determine crystal growth? The triangular ice spike. Am. F. Phys., 66(12), 1041.

Mason, B. J. and J. Maybank. 1960. The fragmentation and electrification of freezing water drops. Q. J. R. Meteorol. Soc., 86(368), 176-185.

Perry, H. F. 1993. Ice spikes — can you explain them? Phys. Teacher, 31 (2), 112.

Perry, H. F. 1995. "Last word" on ice spikes. Phys. Teacher, 33(3), 148-149. 